\title{
EXPLORING THE APPLICATION OF NETWORK ANALYTICS IN CHARACTERIZING A CONCEPTUAL DESIGN SPACE
}

\author{
Gyory, Joshua T.; Goucher-Lambert, Kosa; Kotovsky, Kenneth; Cagan, Jonathan \\ Carnegie Mellon University
}

\begin{abstract}
The ability to effectively analyse design concepts is essential for making early stage design decisions. Human evaluations, the most common assessment method, describe individual design concepts on a variety of ideation metrics. However, this approach falls short in creating a holistic representation of the design space as a whole that informs the underlying relations between concepts. Motivated by this shortcoming, this work leverages network theory to visualize and characterize features of a conceptual design space. To illustrate the utility of network theory for these purposes, a network composed of a corpus of solutions to a design problem and their semantic similarity is derived, and its design properties (e.g., uniqueness and innovation potential) are studied. This network-based approach not only characterizes features of individual designs themselves, but also uncovers more nuanced properties of the design space through studying emerging clusters of concepts. Overall, this work expands on developing research in design, demonstrating the value in applying network analytics to a conceptual design space as an engineering support tool to aid design decision-making.
\end{abstract}

Keywords: Conceptual design, Evaluation, Design methods

\section{Contact:}

Cagan, Jonathan

Carnegie Mellon University

Mechanical Engineering

United States of America

cagan@cmu.edu

Cite this article: Gyory, J.T., Goucher-Lambert, K., Kotovsky, K., Cagan, J. (2019) 'Exploring the Application of Network Analytics in Characterizing a Conceptual Design Space', in Proceedings of the 22nd International Conference on Engineering Design (ICED19), Delft, The Netherlands, 5-8 August 2019. DOI:10.1017/dsi.2019.201 


\section{INTRODUCTION}

Obtaining a design space representation and understanding its properties is critical during conceptualization to weigh possible successes of and comparisons between ideas. In addition to describing features of individual design concepts, it remains especially challenging to understand detailed characteristics of the space of concepts as a whole. Uncovering this information would allow designers to more effectively predict and assess the future potential of design alternatives earlier in the design process. Currently, the most common method for assessing product attributes of early-stage design concepts utilizes expert evaluations. Yet, while the use of human evaluators can describe attributes of individual design concepts, this method falls short in characterizing the entire design space and the interrelations between concepts. To mitigate this shortcoming, ongoing efforts exist to supplement the process of design space exploration with computational tools that not only measure attributes of individual concepts, but also the overall design space they compose (Maher and Fisher, 2012).

This research explores a unique, computational perspective by leveraging the application of network analytics to visualize and characterize a conceptual design space. The versatility of network science in describing and analysing different scales and modalities of data sets, as well as its statistical and graphical underpinnings, open the opportunity for this type of application. More importantly, a network approach supports the ability to uncover these holistic design space properties that emerge when studying clusters of designs and their interconnections. While network theory has seen some applications in engineering research to aid design decisions, this work proposes a novel implementation, employing the generation and evaluation of a semantically-derived, conceptual design space. If design attributes and design space exploration can be linked to corresponding network analytics, network theory could serve as a valuable computational method to support the process of design space assessments, thereby eliminating some of the limitations inherent in current approaches.

\section{BACKGROUND}

\subsection{Network science}

Network science, a subset of graph theory, studies the interrelation of entities via their connectivity (Brandes et al., 2016). These entities and relations take on many different forms, depending on the specific network application. For example, one of the most widely studied types of networks, particularly in recent years, is social networks, which have seen disparate applications ranging from psychology and biology, to information science (Borgatti et al., 2009). In social networks, actors or organizations connect based on their relationships, the existence of knowledge/information sharing and/or direct communication, and how these relations dynamically evolve over time.

Regardless of the specific application, the same underlying theoretic concepts comprise all network structures. Nodes typify actors, and represent individual people, organizations, knowledge, skills, or any key entity characterizing the problem. Edges link discrete nodes, varying by their specified type, such as role-based relations (e.g., friendship), cognitive relations (e.g., transfer of resources), or proximity relations (e.g., physical distances) (Borgatti and Halgin, 2011). Additionally, edges may vary based on their direction, whether the relation is in one particular direction (known as a directed network), or if the direction between connecting nodes does not matter (known as an undirected network). Edges are either binary or weighted. Binary edges only depend on whether a connection between nodes exists or not, typically computationally designated with a 1 or 0 , respectively. A weighted edge depends on the strength of the relation between adjoined nodes, such as the frequency of interaction between actors, how long the interaction exists, or a physical distance. Accordingly, the strength of network theory is in its versatility. Through the use of visual, statistical, and graphical analytics, network theory can be applied to varying scales and types of data sets; in this work, to a set of engineering design concepts.

\subsection{Current methods of assessing design concepts}

In the engineering design literature, human evaluators commonly assess design metrics manually (Fu et al., 2010; Gosnell and Miller, 2015; Gyory et al., 2019; Song, Lopez, et al., 2017b; Toh and Miller, 2016). A variety of different metrics can be assessed including, but not limited to, quality, novelty, quantity, feasibility, and usefulness of design ideas. Relying on human experts requires the evaluators to be trained on the rating specifications of these quantifiable criteria. To eliminate some of the subjectivity in these assessments, normally several evaluators assess a subset of the same 
designs to gain inter-rater agreement, quantified through a correlation test; the intra-class correlation coefficient is a common example. While this process of expert evaluations provides specific metric analysis on individual designs, it does not provide a holistic representation of the entire space and how designs relate to one another. The work presented in this paper utilizes human assessments in a way that is easier and more meaningful to compare across designs and the design space as a whole. A portion of current research has transitioned towards crowdsourcing and computational approaches for executing these design assessments. Crowdsourcing minimizes the workload, especially when rating a large corpus of designs. However, localizing individuals with sufficient levels of expertise remains a fundamental issue, particularly for complicated or nuanced tasks relevant to engineering design (Burnap et al., 2015; Gercer Ulu et al., 2018). Similarly, concerns with trust and quality control exist, as well as the manner in which research investigators frame the task and incentivize their crowd workers. (Kittur et al., 2008; Redi and Povoa, 2014). A more recent computational approach utilizes idea maps to measure similarity and novelty of design concepts (Ahmed et al., 2018). A two-dimensional, visual representation of a design space, an idea map diagrams design similarities derived by triplet queries (i.e. given two design options B and C, the query asks: "Which design is more similar to design A?"). However, idea maps still require human evaluators to execute these queries. The network approach in the current paper eliminates the need of human workers, and instead, computationally determines similarities between design concepts using Latent Semantic Analysis.

\subsection{Applications of network science in engineering design}

Applications of network theory already appear in the field of engineering design, particularly in representing complex engineering systems (Chen et al., 2018). Most recently, Walsh et al. model engineering systems as behavioural networks to study critical design parameters and their effect on the overall failure of systems (Walsh et al., 2018). The work has discovered that bridging nodes in these behavioural networks, nodes which link distinct communities, are vital in understanding how systems fail. Along the same vein, design changes have also been modelled using network theory (Ma et al., 2017). In their work, the mechanical design specifications of systems construct design property networks. By comparing linkage weights, node degrees (number of connections on a node), and chains of links, design changes can be assessed, along with their proliferation and propagation through an engineering system. Sosa et al. also show that in systems with complicated architectures, a correlation exists between the number of hubs in a system (a component with a high degree distribution) and the quality of that component (Sosa et al., 2011). Overall, these works support the advantage of modelling complex engineering systems as networks, alleviating downstream costs, by serving as an early diagnostic tool for system testing.

Network theory has also been utilized in understanding the relationships between consumer behaviour and products (Wang et al., 2015). In that work, interactions such as purchases, buyer considerations, product similarities, and other associations are studied, by generating distinct product and customer network models. Additionally, through the construction of customer demographics and product attribute graphs, network science, as a prognostic tool, forecasts and quantifies the impact of technology on customer behaviours and marketplace competition. (Wang et al., 2016). As such, utilizing network theory to characterize consumer preferences for products has benefited in the support of engineering decision making and the optimization of product attributes.

Network theory has also been applied to understand ideation strategies and design creativity. Song et al., map a patent space into home, near, and far fields, based on knowledge distance, to a particular design task The researchers examine how participants utilize patents from these varying fields as stimuli for ideation, and how these stimuli affect problem-solving outcome (Song, Srinivasan, et al., 2017a). In addition, Ahmed et al., create bisociative information networks to uncover cross-domain inspiration and creativity (Ahmed and Fuge, 2018). Clearly, network science has aided engineers as a support tool in a variety of aspects of early phase design. This paper introduces network science as a means to represent and assess characteristics of a semantic, concept space, generated for a specific design problem. 


\section{METHOLDOGY}

\subsection{Generation of the semantic design space}

The data constructing the network in this work originated from a previous experiment run at Carnegie Mellon University, where 60 undergraduate student designers solved a conceptual engineering design problem (Goucher-Lambert et al., 2019). The problem asked participants to think of solutions that would minimize accidents for people walking and texting on a cell phone (abbreviated). Ten minutes into the problem-solving process, participants typed a functional description of their current design idea. To support their design ideation, participants were then provided with an adaptive, inspirational solution stimulus, either semantically near or semantically far, from their current design concept. For the succeeding ten minutes, participants could further iterate on their current design or move in a completely novel design direction. The semantic distance between their concept and 115 potential example solution stimuli was computed using Latent Semantic Analysis (LSA): a computational approach using singular value decomposition and the cosine similarity to compare textual document vectors (Landauer and Foltz, 1998). Each candidate stimulus that could be provided to participants originated from a set of 115 example solutions to the aforementioned cell phone problem (GoucherLambert and Cagan, 2019). With the aforementioned approach, 29 distinct designs (of the possible $115)$ were computationally designated as semantically near (maximum semantic similarity) or far (minimum semantic similarity) to designers' midpoint solutions, and thus, provided as inspirational stimuli. The remaining 86 designs in the corpus were not provided as stimuli during the study.

The 115 candidate design stimuli formed the design space network visualized and analysed in the current work. Each of the 115 design concepts were composed of a combination of drawings and textual annotations. Three graduate students at Carnegie Mellon (all members of a design methodology research lab), manually transcribed functional descriptions for the concepts (required to be at least 75 words in length to provide a sufficient text length to run LSA). With a textual description for each design concept, the semantic similarity was then computed between all pairs of concepts, resulting in a $115 \times 115$, symmetric, similarity matrix, $S$. In addition to these textual descriptions, human evaluators rated the design concepts on four design metrics: novelty, usefulness, feasibility, and quality.

\subsection{Innovation metric assessment}

In companion work by the authors, a new metric for conceptual design assessment was defined (Goucher-Lambert et al., 2019). The motivation for this new assessment derived from the ambiguity in, and lack of a universal definition for, the commonly referenced design metric quality. The companion work proposed a new, encapsulating metric for the overall goodness of an idea, based on more quantifiable definitions and design attributes. These include the feasibility, usefulness, and novelty of a design. Thus, this metric, $I$, termed the "innovation metric," characterizes the overall goodness of a design solution, defined as follows:

$$
I=F \cdot U+N
$$

where $F$ is the feasibility of a design, $U$ is the usefulness, and $N$ is the novelty. As a preliminary proof to show this new score encapsulated the same attributes as quality, a correlation test was performed between the two (innovation score and quality), across all 115 designs. With a strong correlation ( $r=$ 0.75 ), the analysis validated this new innovation metric sufficiently and robustly represented the overall quality of the concepts. As applied in the current work, the innovation metric was computed for the 115 concepts and added as a supplemental attribute for each design. Moreover, as opposed to quality, this new metric was the metric used to depict the goodness of the design concepts in the network analysis.

\subsection{Design concept network and node attributes}

ORA, a toolkit for dynamic network analysis (Carley, 2014), provided network visualization capabilities to this work. The nodes in the network represented a specific design concept for the problem, each with 6 associated node attributes: feasibility score, usefulness score, novelty score, quality score, innovation score, and stimulus type. External evaluators assessed the feasibility, usefulness, novelty, and quality ratings on a scale from [ 0 - 2], with 0 attributed to designs exhibiting the lowest potential, and 2 attributed to designs exhibiting the highest potential on the respective 
metrics. As a result, the innovation score, encapsulating the overall design "goodness" and innovation potential, ranged from $[0-6]$. The stimulus type attribute identified whether the design was provided as a semantically 'near' or semantically 'far' stimulus (or as 'none,' if not provided as a stimulus during the study previously discussed).

The edges connecting design concepts, weighted by the semantic similarity, existed between all pairs of nodes. Thus, via network science nomenclature, the network topology was fully connected. These edges were also undirected, meaning that the direction of the link connections does not matter (i.e., if design A has a semantic similarity of 0.48 with design $\mathrm{B}$, the same is true in both directions: A to B and B to A). The semantic link values, derived from the cosine similarity between two vectors, varied between 0 and 1. Altogether, the network, fully-connected and undirected, contained 115 nodes and 13,225 links.

\subsection{Network centrality}

One of the most powerl graph properties, network centrality, captures the key actors (i.e., nodes) in a network structure (Freeman, 1978). Nodes designated as key actors have the most connections, have the most control on the flow of information, and have the most overall influence on the network. Separate measures of centrality impose different conceptual interpretations depending on the specific network application (Opsahl et al., 2010). In order to understand where in the design space distinct concepts lie relative to one another, this work will focus on closeness centrality. Closeness centrality determines the sum of shortest distances from one node to all other nodes within a network, mathematically formulated as follows:

$$
C_{c}\left(n_{j}\right)=\left[\sum_{j=1}^{g} d\left(n_{i}, n_{j}\right)\right]^{-1},
$$

where $d\left(n_{i}, n_{j}\right)$ defines a distance function from node $n_{i}$ to node $n_{j}$, with $g$ nodes in the network (Wasserman and Faust, 1994). The inverse in the formulation guarantees the conceptual interpretation between closeness and distance. However, because the semantic similarity already corresponds to a measure of closeness, the distance metric between any two nodes, $d\left(n_{i}, n_{j}\right)$, should be the inverse of the link values in the current network. That is, higher semantic similarity between two nodes corresponds to a smaller distance. To correct this analytically, the distance is redefined to be the inverse of the semantic similarity, $s$, between two nodes (node $n_{i}$ to node $n_{j}$ ) in the network:

$$
C_{c}\left(n_{j}\right)=\left[\sum_{j=1}^{g} \frac{1}{s\left(n_{i}, n_{j}\right)}\right]^{-1} .
$$

\section{RESULTS}

Recent work have focused on how different distances of inspirational stimuli impact design ideation. As mentioned, a subset of the underlying designs in the corpus were used specifically for this purpose: provided as a near or far stimulus to a designer's current concept to influence design outcomes. To better grasp the characteristics of these stimuli and how they relate to one another within a bounded design space, the analyses in this section focused on these designs. To facilitate with this examination, a sub-network, containing only the semantically near and far stimuli, was extracted from the broader network. The sub-network contained 15 designs provided as near stimuli and 14 designs provided as far stimuli, and thus, the sub-network totalled 29 distinct concepts.

\section{1 Network density}

Before analysing the more intricate network characteristics, a representation of the complete network space was gained by first understanding a global property, the network density $(\rho)$ : the proportion of links that exist to the total number of possible links. The network density provided a global spectrum of similarity values across the entire design space. However, visualizing the original, fully connected network did not contribute meaningful insight, because with the density being $\rho=1$, all possible connections existed. Therefore, in order to start uncovering interesting characteristics regarding 
specific design clusters within the network, it was necessary to raise the threshold on the link connections. This enabled the most dissimilar (low cosine similarity) designs to become disconnected from the network, and facilitated visualizing and revealing the strongest interconnections within the design space. Figure 1 shows the network density for the entire network as a function of increasing link weights. In total, the network contained 13,225 links, with a mean semantic similarity of $\bar{s}=0.49 \pm 0.08$ ( \pm 1 standard deviation $)$. The network density sharply decreased between a narrow similarity interval $(s \approx 0.41$ and $s \approx 0.57)$. Ideally, the design space would include a large range of values, with highly similar and highly dissimilar designs, in order to effectively study clusters of concepts. For example, if all concepts in a design space were all the same distance semantically, no meaningful groupings of concepts would materialize. Even though this design space does not necessarily contain the most diverse set of solutions possible, the concepts were still distinct enough to gain constructive insights.

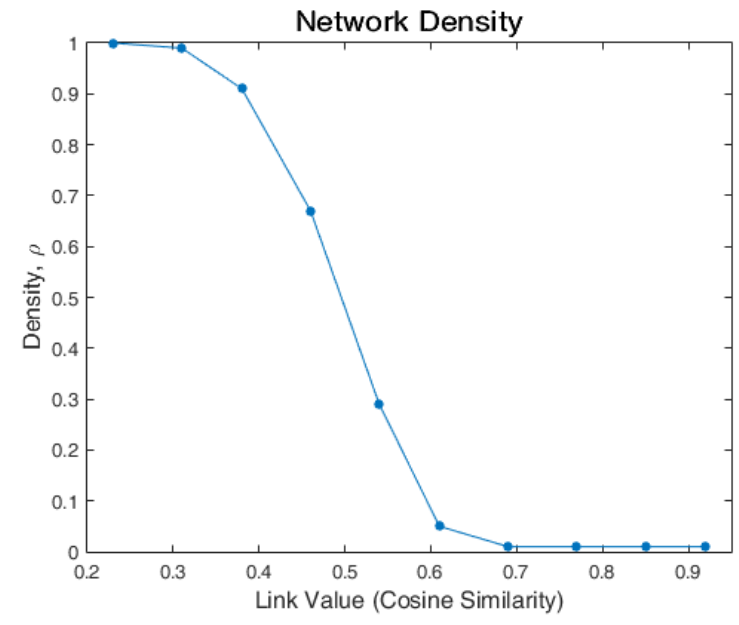

Figure 1. Overall network density at increasing link values (i.e. semantic similarity), accounting for all 13,225 connections

\subsection{Design stimuli - examination of closeness centrality}

In correspondence with the density, to better visualize the broader network, the link threshold was raised to $s=0.64$. Therefore, all pairs of designs with a semantic similarity less than this threshold become disconnected from each other in the visualization. It should be noted that this exact threshold value was arbitrarily chosen and bore no impact on the current analysis (this being one of the highest thresholds before the majority of stimuli broke apart from each other in the graph). Figure 2 shows the designs coloured based on their stimulus type. Red nodes indicate semantically "far" stimuli, blue nodes indicate semantically "near" stimuli, and the remaining nodes (green), indicate nodes not provided as stimuli during the experiment.

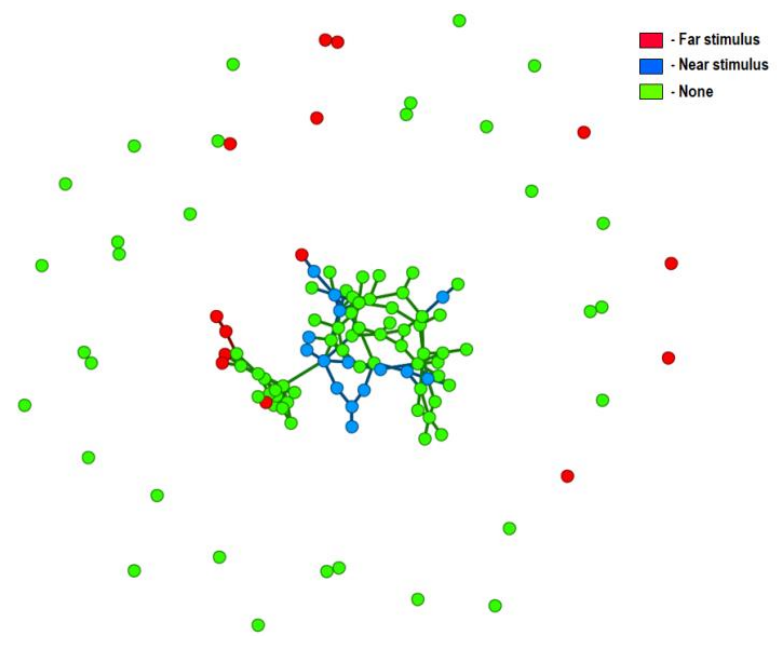

Figure 2. Network visualization of concept design space coloured via stimulus type, with the similarity threshold set at, $s=0.64$ 
Based only on graphical inspection of the network, semantically near stimuli already appeared to be more central and deeply interconnected within the overall network. Even at a similarity threshold of $s$ $=0.64$, all near stimuli remained linked, while 57\% (8 of 14) of the far stimuli had split off from the central group. Even those far stimuli remaining in the central group were loosely coupled, on the verge of splitting off. Now to analytically assess whether the near stimuli were actually more central relative to the far stimuli, the closeness centrality (the inverse of the relative distance from a node to all other nodes within a network) was calculated for the two stimulus types, with results shown in Figure 3. The semantically near stimuli exhibited a significantly higher average closeness centrality than the semantically far stimuli $(p<<0.01, f=0.91)$, indicating the connectedness and thus commonality of these designs within the network.

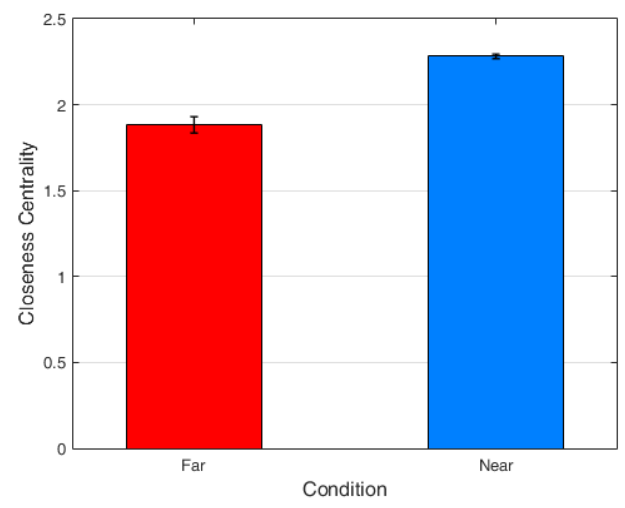

Figure 3. Average closeness centrality for semantically near and far stimuli. Error bars show \pm 1 S.E.

\subsection{Design innovation - understanding design space topology}

The 29 design stimuli were then extracted from the overall network into their own sub-network, and coloured based on their innovation score to visualize the goodness of the concepts. To uncover the emergence of design clusters, 6 distinct snapshots depicted the network at incremental similarity thresholds, starting at $s=0.30$, to $s=0.45, s=0.50, s=0.55, s=0.60$, and $s=0.64$ (Figure 4). The design innovation score (calculated by Equation 1) ranged from $0-6$, with lower scores shown by cold colours (dark blue: $I=0$; green: $I=2,3$ ), and higher scores shown by the hotter colours (yellow: $I=4$, orange: $I=5$; red: $I=6$ ).

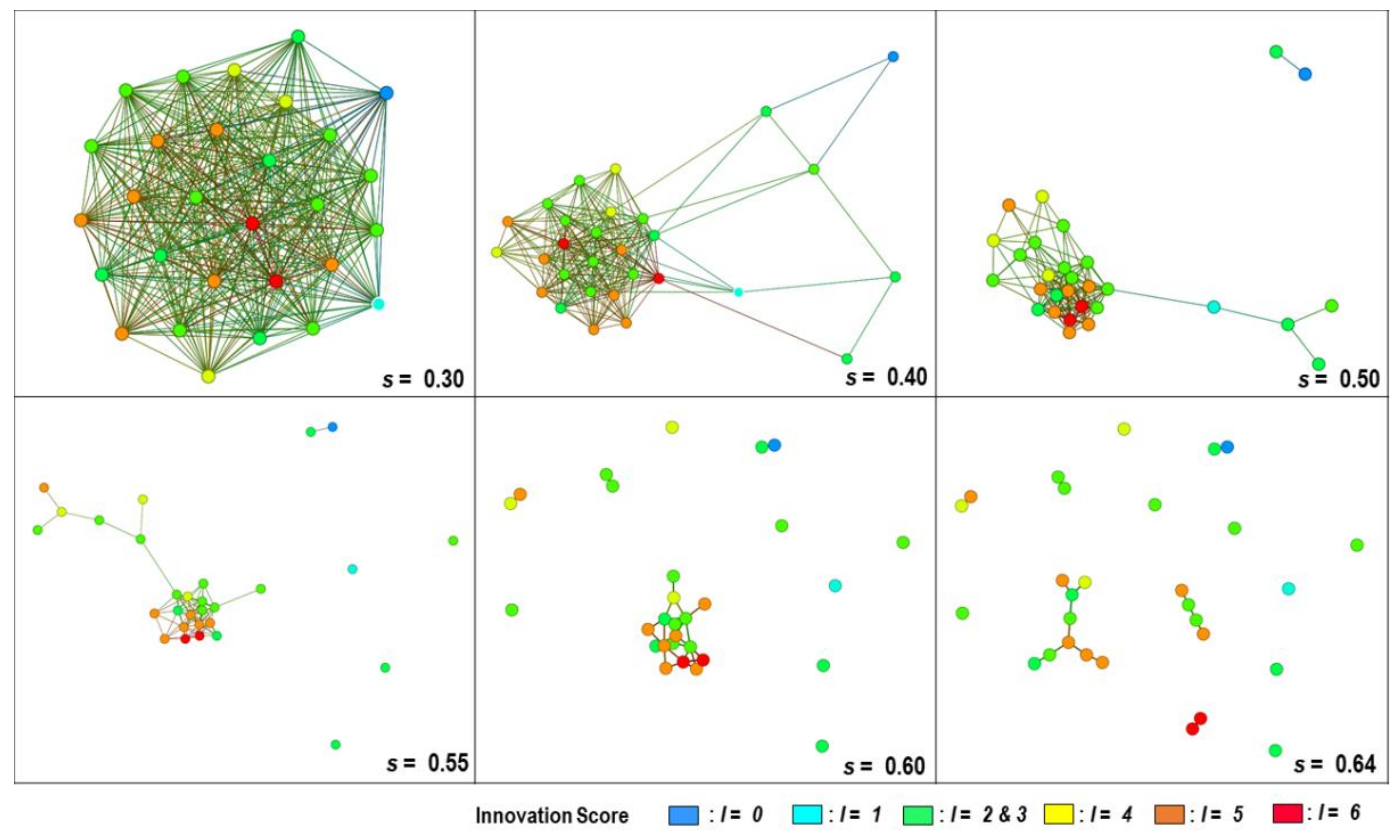

Figure 4. The 29 design stimuli, shown at 6 different similarity thresholds, and coloured based on their innovation score, I 
As seen through the evolution of the network in Figure 4, qualitatively, as the similarity threshold increases, designs with a higher innovation score tend to remain more densely connected within the sub-network. Furthermore, designs with similar innovation scores tend to be more semantically similar with each other. At a threshold of $s=0.64$ (lower, right hand side of Figure 4), designs with innovation scores of $I=4, I=5$, and $I=6$ (yellow, orange, and red, respectively), still remain clustered, especially with each other. This evolution provides insight into the topology of the design space: the location of the clusters of good and bad designs, and the strong semantic relations between them.

\section{DISCUSION}

By examining a pre-populated network composed of problem solutions, this works takes a network analytics perspective to characterize features of a design space. A subset of the 115 designs (29 designs, specifically) are used in a previous experiment as inspirational stimuli. Consequently, the design space in this work presents an opportunity to study properties of these semantically near and far stimuli embedded within the broader corpus. A preliminary, visual inspection of the network graph shows that semantically near stimuli are more central and connected compared to far stimuli. In and of itself, this result is intriguing, as there is not necessarily an expectation that the semantically near stimuli (each independently adapted to individual problem solvers) should all be more central and coupled with each other compared to far stimuli. Thus, in general, the far stimuli appear to be relatively more unique designs in this pre-populated design space. Whether this finding remains true of only this particular problem domain and/or pre-defined design spaces, or is an inherent property of all sets of near and far stimuli in general, requires future studies to thoroughly address.

Now that the near and far stimuli appear to occupy different levels of uniqueness within the concept space, the next analysis explores whether a network metric, closeness centrality, can accurately assess and link to this design metric (uniqueness). Closeness centrality calculates how central nodes are based on their cumulative distance from a respective node to all others within a graph. Conceptually, if near stimuli are truly less unique designs within the design space (network), these designs should have a shorter distance (i.e., higher similarity) to all other designs in the network, and consequently, a higher centrality. Computing the closeness centrality metric for nodes of each stimulus type reveals that near inspirational stimuli do possess a higher closeness centrality than far inspirational stimuli. In other words, near stimuli are more closely related and connected to all other designs within the design space, relative to far stimuli.

An interesting supplementary investigation compares the above computation to the novelty metric of these stimuli characteristics. Recall that human evaluators independently assessed the concepts based on their novelty. In the design literature, it is common to define the novelty of a design based upon its uniqueness within a design space (Verhaegen et al., 2012). Therefore, one would assume that if the near designs are not particularly unique (as illustrated by the preceding analysis of closeness centrality), then they should also display low ratings for novelty. By comparing the average novelty ratings for each of the two experimental conditions, the near stimuli, unexpectedly, possess significantly higher novelty scores compared to the far stimuli (near: $\mu=1.4$, far: $\mu=0.9 ; p<0.02, f=$ 0.39). This finding is an illustrative example of a point of contention within the design community; novelty may actually encapsulate more than a design's rarity (i.e., uniqueness) in a design space.

This paper also analyses the goodness of the design stimuli and uses this property to visualize the topology of the design space. Here, an original, single measure defines the overall goodness of each stimulus (innovation metric, $I$ ), derived and calculated based on the feasibility, usefulness, and novelty of each design concept (Equation 1). Even though external evaluators independently assess these three measures, the innovation metric represents a significantly more objective approach to assess the overall innovative potential of a design, as opposed to assessing quality, which possess no real tangible or universal definition among design researchers. Furthermore, in the way that this new innovation metric is formulated, it exhibits a high correlation with quality $(r=0.75)$, validating the applicability of this metric for representing overall goodness in this work.

The 29 near and far stimuli are then visualized with this innovation metric. Due to the density of the links, the stimuli are extracted into their own sub-network and visualized at incrementally increasing similarity thresholds (Figure 4). While all stimuli interconnect at a low threshold, $s=0.30$, raising this value qualitatively reveals that good designs, in terms of the innovation metric, $I$, are drawn towards 
each other (i.e. are also more semantically related). Even at a fairly high similarity, $s=0.64$, designs with higher innovation scores remain semantically connected, while those designs with low innovation scores become disconnected (or stay connected with other low I designs). This result has several implications for design. The visualization alone reveals that several clusters of "good" designs emerge. This can be utilized as a visual support method, aiding designers with a graphical view of the topology of the design space: how innovative and non-innovative designs compare to each other. Additionally, with the power of network analytics, these clusters can be computationally and automatically demarcated via clustering algorithms, left here for future work. The high/low innovation clusters also emerge with high degrees of semantic similarity. This opens the prospect of leveraging a network framework as a prognostic tool for design support. For example, if a designer generates a new design concept (not already in the design space), with a textual description, they could, in theory, map their new design into this semantic design space to determine which design(s) is (are) most similar. Because each design node possesses individual design metrics (node attributes), the attributes of a new design could be automatically predicted/generated.

While the network employed in this work studies a conceptual design space, the methods and implications in this paper are not necessarily limited to this. For example, the general approach of network analytics can be applied to a wide range of design processes consisting of an entity (here, design concepts) and some underlying relation linking them (here, semantic similarity). The study of such interactional phenomena lies at the core of engineering, thereby making a network perspective an advantageous approach.

\section{CONCLUSION}

The ability to effectively analyse design concept attributes is critical in the early stages of the product development process. Often resource intensive, current methods via human evaluators and crowdsourcing possess inherent difficulties, and lack in providing a comprehensive representation of the design space as a whole. Consequently, a need exists to automate the process of design exploration and to develop computational tools to help assess design concepts and their relational properties. This work expands research on the growing implementation of network theory in engineering design to aid early-stage decision making. The motivation of utilizing network theory originates from its statistical groundings, graphical power, and ability to study the relational properties between entities.

This work presents the representation of a semantically-derived conceptual design space as a network problem. In the resulting graphs, nodes denote individual design concepts, linked via their semantic similarity between each other. The paper explores the use of network analytics to characterize different features of this semantic design space, and specifically, near and far inspirational stimuli. Closeness centrality, a common network metric, substantiates the uniqueness (i.e., relative similarity between concepts within a pre-defined space) of designs within the network. Additionally, closeness centrality illustrates the difference between novelty and uniqueness of design concepts - suggesting that, perhaps, uniqueness is a necessary but not sufficient condition of novelty. Through a new and objective innovation metric, $I$, the overall goodness of design concepts is then visualized within the space. Distinct clusters of good designs emerge with high levels of similarity, providing a topological view of the design space. Overall, this work illustrates the application of network science as a promising computational approach and graphical support tool for characterizing design attributes, and more critically, for providing a holistic representation of the entire conceptual design space.

\section{REFERENCES}

Ahmed, F. and Fuge, M. (2018), “Creative Exploration Using Topic Based Bisociative Networks”, Design Science, Vol. 4 No. 12, pp. 1-30.

Ahmed, F., Fuge, M., Hunter, S. and Miller, S. (2018), "Interpreting Idea Maps : Pairwise comparisons reveal what makes ideas novel", Journal of Mechanical Design.

Borgatti, S., Mehra, A., Brass, D.J. and Labianca, G. (2009), "Network Analysis in the Social Sciences", Science, Vol. 323 No. April, pp. 892-896.

Borgatti, S.P. and Halgin, D.S. (2011), “On Network Theory”, Organization Science, Vol. 22 No. 5, pp. 11681181.

Brandes, U., Robins, G., Mccranie, A.N.N. and Wasserman, S. (2016), "Science : What is network science?", Vol. 1 No. April 2013, pp. 1-15.

Carley, K.M. (2014), “ORA - A Toolkit for Network Analysis and Visualization”. 
Chen, W., Heydari, B., Maier, A.M. and Panchal, J.H. (2018), "Network-based Modeling and Analysis in Design", Design Science, Vol. 4, p. e16.

Freeman, L.C. (1978), “Centrality in Social Network Conceptual Clarification”, Social Networks, Vol. 1 No. 3 , pp. 215-239.

Fu, K., Cagan, J. and Kotovsky, K. (2010), "Design Team Convergence: The Influence of Example Solution Quality”, Journal of Mechanical Design, Vol. 132 No. 11, p. 111005.

Gercer Ulu, N., Messersmith, M., Goucher-Lambert, K., Cagan, J. and Kara, L.B. (2018), "Wisdom of MicroCrowds in Evaluating Solutions to Esoteric Engineering Problems", Journal of Mechanical Design.

Gosnell, C.A. and Miller, S.R. (2015), "But Is It Creative? Delineating the Impact of Expertise and Concept Ratings on Creative Concept Selection”, Journal of Mechanical Design, Vol. 138 No. 2, p. 021101.

Goucher-Lambert, K. and Cagan, J. (2019), "Crowdsourcing Inspiration: Using crowd generated inspirational stimuli to support designer ideation”, Design Studies, Elsevier Ltd., Vol. 61, pp. 1-29.

Goucher-Lambert, K., Gyory, J.T., Cagan, J. and Kotovsky, K. (2019), “Computationally Adaptive Stimuli for Real-Time Design Support”, (Working Paper).

Gyory, J.T., Cagan, J. and Kotovsky, K. (2019), "Are you better off alone? Mitigating the underperformance of engineering teams during conceptual design through adaptive process management", Research in Engineering Design, Springer London, Vol. 30 No. 1, pp. 85-102.

Kittur, A., Chi, E.H. and Suh, B. (2008), "Crowdsourcing User Studies With Mechanical Turk", CHI '08 Proceedings of the SIGCHI Conference on Human Factors in Computing Systems, pp. 453-456.

Landauer, T. and Foltz, P. (1998), “An introduction to latent semantic analysis", Discourse Processes, Vol. 25, pp. 259-284.

Ma, S., Jiang, Z. and Liu, W. (2017), “A design change analysis model as a change impact analysis basis for semantic design change management", Proceedings of the Institution of Mechanical Engineers, Part C: Journal of Mechanical Engineering Science, Vol. 231 No. 13, pp. 2384-2397.

Maher, M. and Fisher, D. (2012), "Using AI to evaluate creative designs", Proceedings of the 2nd International Conference on Design Creativity, No. September, pp. 45-54.

Opsahl, T., Agneessens, F. and Skvoretz, J. (2010), "Node centrality in weighted networks: Generalizing degree and shortest paths", Social Networks, Elsevier B.V., Vol. 32 No. 3, pp. 245-251.

Redi, J. and Povoa, I. (2014), "Crowdsourcing for Rating Image Aesthetic Appeal", Proceedings of the 2014 International ACM Workshop on Crowdsourcing for Multimedia - CrowdMM '14, pp. 25-30.

Song, B., Srinivasan, V. and Luo, J. (2017a), "Patent stimuli search and its influence on ideation outcomes", Design Science, Vol. 3, p. e25.

Song, H.I., Lopez, R., Fu, K. and Linsey, J. (2017b), "Characterizing the Effects of Multiple Analogs and Extraneous Information for Novice Designers in Design-by-Analogy”, Journal of Mechanical Design, Vol. 140 No. 3, p. 031101.

Sosa, M., Mihm, J. and Browning, T. (2011), "Degree Distribution and Quality in Complex Engineered Systems", Journal of Mechanical Design, Vol. 133 No. 10, p. 101008.

Toh, C.A. and Miller, S.R. (2016), "Creativity in design teams: the influence of personality traits and risk attitudes on creative concept selection”, Research in Engineering Design, Springer London, Vol. 27 No. 1, pp. 73-89.

Verhaegen, P.-A., Vandevenne, D. and Duflou, J.R. (2012), "Originality and Novelty: A different Universe”, International Design Conference - Design 2012, Dubrovnik - Croatia, pp. 1961-1966.

Walsh, H.S., Dong, A. and Tumer, I.Y. (2018), "The role of bridging nodes in behavioral network models of complex engineered systems", Design Science, Vol. 4, p. e8.

Wang, M., Chen, W., Huang, Y., Contractor, N.S. and Fu, Y. (2015), “A Multidimensional Network Approach for Modeling Customer-Product Relations in Engineering Design”, ASME 2015 International Design Engineering Technical Conferences and Computers and Information in Engineering Conference, Boston, Massachusetts, USA, available at: https://doi.org/10.1115/DETC2015-46764.

Wang, M., Sha, Z., Huang, Y., Contractor, N., Fu, Y. and Chen, W. (2016), "Forecasting Technological Impacts on Customers' Co-Consideration Behaviors: A Data-Driven Network Analysis Approach", ASME 2016 International Design Engineering Technical Conferences and Computers and Information in Engineering Conference, Charlotte, North Carolina, p. V02AT03A040.

Wasserman, S. and Faust, K. (1994), Social Network Analysis: Methods and Applications, Cambridge University Press.

\section{ACKNOWLEDGMENTS}

This work was supported by the Air Force Office of Scientific Research (AFOSR) under grant No. FA9550-18-1-0088. Any opinions, findings, and conclusions or recommendations expressed in this paper are those of the authors and do not necessarily reflect the views of the sponsors. 